\title{
Cloud-RAN Factory: Instantiating virtualized mobile networks with ONAP
}

\author{
Veronica Quintuna Rodriguez, Romuald Corbel, Fabrice Guillemin, Alexandre Ferrieux \\ Orange Labs, 2 Avenue Pierre Marzin, 22300 Lannion, France \\ \{veronica.quintunarodriguez, romuald.corbel, fabrice.guillemin, alexandre.ferrieux\}@orange.com
}

\begin{abstract}
In this demo, we exhibit the negotiation based on the TM Forum framework (Customer Facing Service and Resource Facing Service) and the deployment of a fully virtualized end-toend mobile network (including a RAN desegregated into Remote Unit, Distributed Unit and Centralized Unit) by using ONAP, an open-source network automation platform. The various components of mobile network are containerized and deployed by ONAP on top of Kubernetes. The demo is the first illustration of an end-to-end mobile network, which is fully virtualized up to the remote unit, whose architecture is compatible with the Open-RAN framework, and which implements a PHY layer on the basis of 3GPP 7.3 functional split in Open Air Interface code.
\end{abstract}

Keywords: Cloud RAN, functional split, 5G, NFV.

\section{INTRODUCTION}

In the context of telecommunication networks, virtualization techniques have enabled the emergence of Network Function Virtualization (NFV), which radically changes the architecture and the operation of networks [1], [2]. NFV greatly impacts specific data plane functions such as Firewalls, Deep Packet Inspection, etc. but also core control functions of mobile networks, for instance via the development of virtual Evolved Packet Core (vEPC) in 4G and boosting the design of a completely new control architecture in 5G (Service Based Architecture, SBA). NFV now also applies to more latency sensitive functions such as those of Radio Access Network (RAN), The development of Software Defined Radio (SDR) is long lasting task and the $4 \mathrm{G}$ Open Air Interface (OAI) software suite (including RAN and core network) is today sufficiently stable to run field trials in the context of $\overline{\mathrm{NFV}}$ and offers a development platform to investigate new features of RAN] [3]; the development of an $5 \mathrm{G} \mathrm{OAI}$ is also rapidly progressing.

The emergence of NFV and its application to core and RAN has boosted the development of various open source communities as Open-RAN (ORAN), Open Network Automation Platform (ONAP) Open Core Network (OCN), among others. The automation of the deployment of Virtualized Network Functions (VNFs) and their life-cycle management is addressed by the ONAP community. The ONAP platform is roughly based on two main phases: the design time and the run time. Each phase is composed of a number of modules in charge of performing specific tasks. The onboarding and the deployment of a vEPC using ONAP has notably been addressed in [4] in the context of network slicing.

ORAN community [5] addresses more specifically the RAN by specifying the basic building blocks of an open RAN, the interfaces between these blocks as well as the methods of splitting RAN functions. ORAN also introduces various controllers (e.g., RAN Intelligent Controller (RIC) to optimize the RAN performance. In this paper, we shall focus on functional blocks along the data path: Remote Unit (RU) Distributed Unit (DU) and Central Unit (CU) This various modules execute the RAN functions: PDCP, RLC, MAC, and PHY functions. ORAN also considers the orchestration of RAN functions; either in cooperation with ONAP or via a standalone orchestration platform.

It is worth nothing that the desegregation and the softwarization of RAN functions have been used to define open architectures and to implement cloud-native RAN functions under the concept of Cloud-RAN (C-RAN) or Cloud-RAN, see for instance [6], [7].

The cloudification of RAN functions has first considered "high" functions (PDCP and a part of RLC functions to centrally manage the $\mathrm{X} 2$ interface). Those functions are usually implemented in the $\mathrm{CU}$ functional block.

In [7], [8], the virtualization of PHY functions (notably the channel encoding and decoding function) has been implemented by taking benefit of the parallelization by means of thread-pool mechanisms. An implementation in OAI RAN code has shown that it is possible to significantly reduce the execution time of coding/decoding and to increase the distance between the intra-PHY functions (channel coding and MAC functions on the one hand and the rest of PHY functions on the other hand). Channel Coding and MAC functions are now executed in the $\mathrm{DU}$ functional block. $\mathrm{CU}$ and $\overline{\mathrm{DU}}$ functional blocks can be implemented in Virtual Machines managed by OpenStack or Containers managed by Kubernetes.

In this demo, we go one step further by proposing to virtualize the RU functional block. The rationale motivating this approach is that physical infrastructures hosting hardware to transmit and receive radio signals (antenna, power supply, etc.) are more and more equipped with mini data centers, which can virtualized. In that case, an operator can push its own RU software into such data centers and the physical site can then be shared by several operators. This is perfectly in line with the rapidly emerging market of TowerCos and with the need for rationalizing the installation of infrastructures hosting antennas in order to reduce CAPEX.

The proposed demo shows how a fully virtualized technical 
chain corresponding to a mobile network (including RAN and core control) can be instantiated by ONAP on a multicloud platform, i.e., supporting Kubernetes and Openstack as Virtualised Infrastructure Managers (VIMs)

The organization of this demo paper is as follows: In Section [I] we introduce the various elements of the demo and in Section III we describe what is shown by the demo. Some concluding remarks are presented in Section IV

\section{Elements OF THE DEMO}

The objective of the demo is to show how a fully virtual mobile technical chain (including the core control network and the RAN can be negotiated between a customer and the network and automatically instantiated by ONAP The various elements involved in the demo are presented in Figure 1 . The negotiation is performed through the Customer Facing Service (CFS)

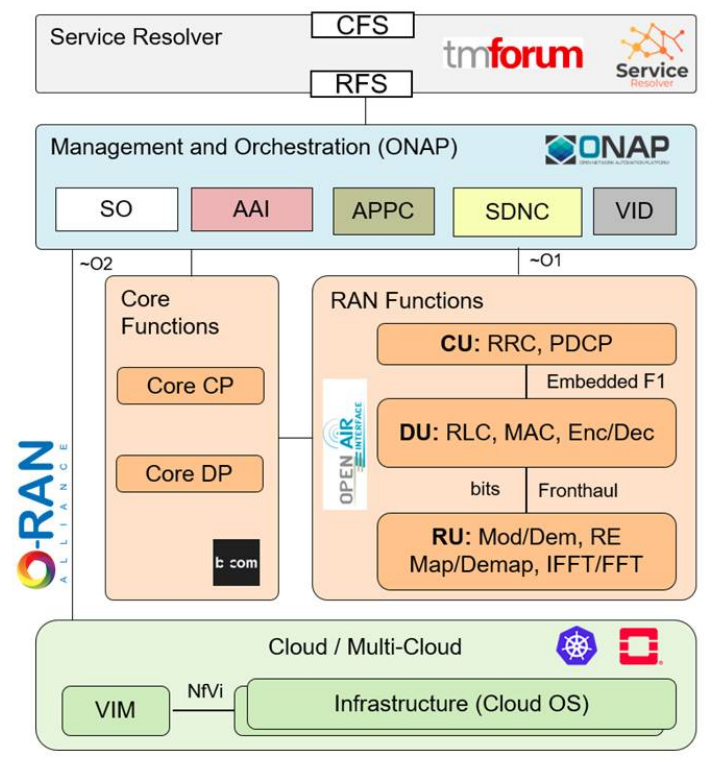

Fig. 1. Elements of the demo according to O-RAN and ODA architectures.

The technical chain is called a network service in the sense of ONAP it is worth noting that such a network service can itself be composed of several network services (e.g., one for the control plane, another one for the core data plane and a last one for the RAN. The negotiation between the customer and the network is performed through the CFS as specified by the TM Forum [9]. The service template chosen via the CFS is then translated by the Service Resolver into a resource request via the Resource Facing Service (RFS), this latter request is then passed to the ONAP platform.

The ONAP platform embeds all the virtualized components of the desired network services. These various components have been previously onboarded during the design time in the form of VMs for the core and containers for the RAN network functions.

ONAP is composed of several modules where the most relevant in this demo are the Service Orchestrator (SO) to deploy the various CNFs, the SDN Controller to deals with the various VNFs and the Active and Available Inventory (AAI) that provides real-time views of available Resources and Services and their relationships. Service resolver consumes AAI information by means of the Northbound Interface (NBI) module.

The cloud platform hosting the VNFs/CNFs is based on OpenStack/Kubernetes. The interface between the orchestrator and the cloud platform is similar to the $\mathrm{O} 2$ interface specified by ORAN and the one between the orchestrator and the RAN functions to the $\mathrm{O} 1$ interface (see [10]).

The functions contained in the $\mathrm{RU}, \mathrm{DU}$ and the $\mathrm{CU}$ are based on OAI code. While ORAN is based on the 7.2 functional split (in the terminology of 3GPP [11]), for separating the low PHY functions, we have here developed, as detailed in [12], the 7.3 split in OAI code. This split consists of transmitting hard bits between the encoder (after puncturing) and Modulation functions in the downlink instead of compressed I/Q signals, and soft bits in the uplink. It is worth noting that in the 3GPP specifications [10], the 7.3 functional split was only considered in the downlink. In the implementation presented in [12], it applies in both downlink and uplink, as illustrated in Figure 2.

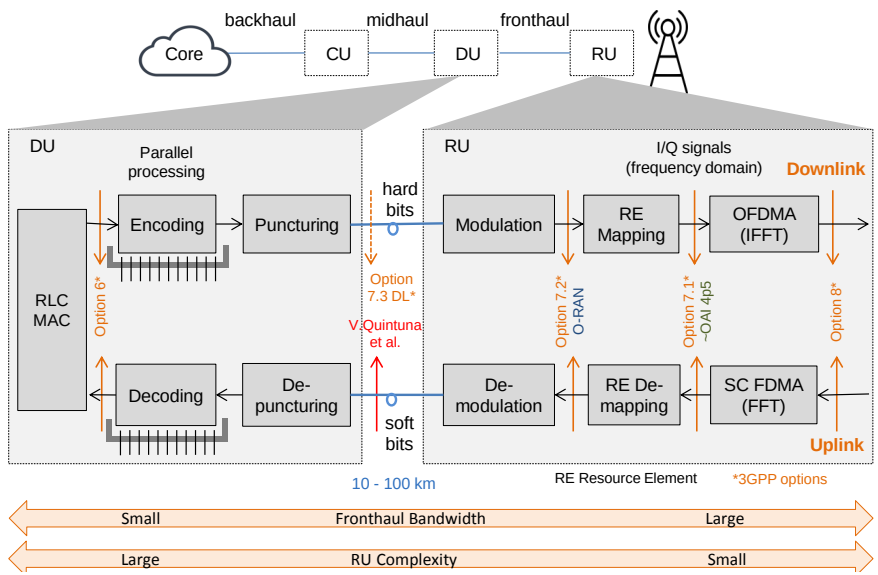

Fig. 2. Implemented functional split architecture.

The 7.3 functional split has been developed because it requires less fronthaul capacity (bandwidth between $\mathrm{RU}$ and DU than 7.2 split. Moreover, the parallelization of coding/decoding code blocks significantly reduces the execution of these tasks, which in turn allows a larger distance between $\mathrm{RU}$ and DU.

In this demo the $\mathrm{DU}$ and $\mathrm{CU}$ have been collocated and deployed as a single container, hence the F1 interface is embedded. Globally, the demo implements on the basis of OAI code a mobile technical chain, which is compatible with the ORAN architecture for the data plane. The various containers executing the RAN and control plane functions are instantiated by means of ONAP. The interfaces between the cloud platform and the SO as well as that between SDNC and the VNFs are 
similar to the $\mathrm{O} 1$ and $\mathrm{O} 2$ interfaces introduced by ORAN, respectively. Thus, the demo is a first illustration of an ORAN compatible end-to-end mobile network orchestrated by ONAP.

\section{WHAT IS SHOWN BY THE DEMO}

The demo realizes the global picture given by Figure 3 The customer negotiates with the network the setup of a virtual end-to-end mobile network tailored to some specific requirements (number of customers, bandwidth, etc.). The request is then passed via the CFS to the Service Resolver, which subsequently translates it into a resource request via the RFS. The resource request in terms of VNFs/CNFs (with associated CPU and storage requirements), bandwidth and connectivity (including the radio coverage) is then handled by ONAP, which deploys the VNFs of the core control and data planes on a Openstack tenant as well as the containerized RU. DU and DU units on a Kubernetes cluster, this latter formed by a remote node hosting the RU. For the core control plane, we have used the Wireless Edge Factory (WEF) developed by $\mathrm{b}<>$ com; this a completely virtualized core network (full $4 \mathrm{G}$ and preliminary $5 \mathrm{G}$ ); see [4] for details in the context of network slicing.

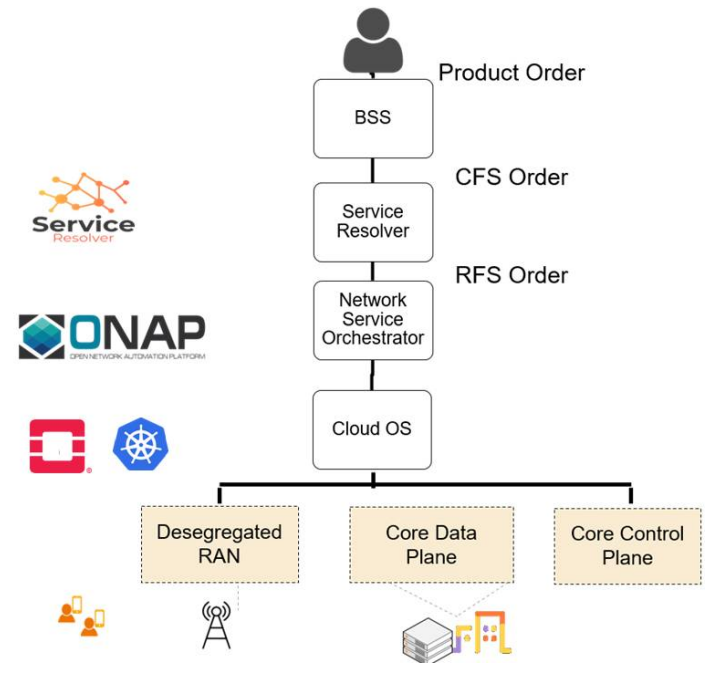

Fig. 3. Cloud-RAN from negotiation to instantiation.

The ONAP GUI is used to verify the deployment of the various VNFs and the associated interconnection network. Figure 4 is a snapshot of the OANP GUI showing the deployment of the elements of the $4 \mathrm{G}$ control plane of the WEF and their internal interconnections.

Once the end-to-end virtualized mobile network is deployed, we verify that the connectivity is really offered by attaching a commercial UE. Figure 5 illustrates the monitoring interface provided by the OAI software, namely the monitoring of the Mobility Management Entity (MME) which shows the successful connectivity.

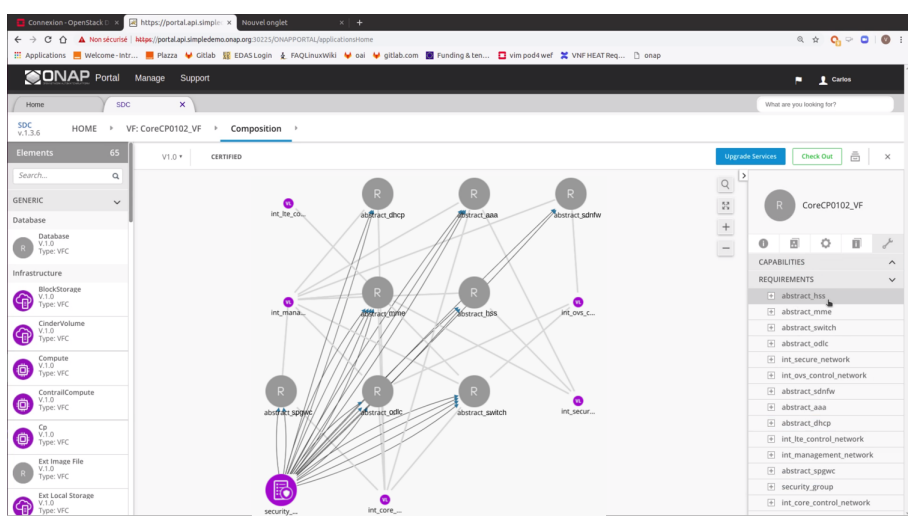

Fig. 4. ONAP GUI showing the deployment of the $4 \mathrm{G}$ WEF components.

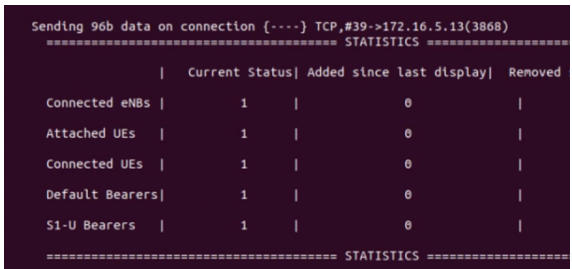

Fig. 5. Monitoring interface of the OAI MME VM.

\section{CONCLUSION}

The demo illustrates the negotiation and the instantiation of an end-to-end mobile network by using the TMF specifications for the negotiation and ONAP for the deployment. The RAN (involving the RU, DU and $\mathrm{CU}$ ) is entirely containerized and deployed by using K8s. The used PHY layer is the 7.3 split implemented in OAI code. The demo is the first realization of a fully end-to-end virtual mobile network compatible with the ORAN architecture and deployed by ONAP.

\section{REFERENCES}

[1] ETSI-NFV, "Network Functions Virtualisation, Architectural Framework," Technical Report ETSI GS NFV 002 V1.1.1, Tech. Rep., 2013.

[2] B. Han, V. Gopalakrishnan, L. Ji, and S. Lee, "Network function virtualization: Challenges and opportunities for innovations," Communications Magazine, IEEE, vol. 53, no. 2, pp. 90-97, 2015.

[3] N. Nikaein, R. Knopp, F. Kaltenberger, L. Gauthier, C. Bonnet, D. Nussbaum, and R. Ghaddab, "Openairinterface 4G: an open LTE network in a PC," in International Conference on Mobile Computing and Networking, 2014.

[4] V. Quintuna Rodriguez, F. Guillemin and A. Boubendir, "5g e2e network slicing management with onap," in 2020 23rd Conference on Innovation in Clouds, Internet and Networks and Workshops (ICIN), 2020, pp. 8794.

[5] O-RAN Alliance, "Operator Defined Next Generation RAN Architecture and Interfaces," https://www.o-ran.org/ [Accessed February-2020].

[6] China Mobile Research Institute, "C-RAN, the road towards green RAN White Paper," 2011.

[7] V. Quintuna Rodriguez and F. Guillemin, "Cloud-RAN Modeling Based on Parallel Processing," IEEE Journal on Selected Areas in Communications, vol. 36, no. 3, pp. 457-468, March 2018.

[8] — - "Contribution to the design and the implementation of a cloud radio access network," 2019.

[9] tmforum, "TMF641 service ordering API user guide v4.0.1," May 2020.

[10] O-RAN Fronthaul Working Group, "Control, User and Synchronization Plane Specification,” O-RAN, Specification, 2019. 
[11] 3GPP, 3rd Generation Partnership Project, Study on new radio access technology Radio access architecture and interfaces, 3 2017, TR 38.801 V14.0.

[12] V. Quintuna, A. Ferrieux, F. Guillemin and L. Thomas, "Cloud-RAN functional split for an efficient fronthaul network," in 2020 International Wireless Communications and Mobile Computing (IWCMC), 2020, pp. 245-250. 